\title{
Employment Status, Readmission and Mortality After Acute Exacerbation of COPD
}

\author{
Peter Ascanius Jacobsen (iD) ${ }^{1,2}$ \\ Kristian Hay Kragholm ${ }^{2,3}$ \\ Christian Torp-Pedersen ${ }^{4}$ \\ Daisy JA Janssen ${ }^{5,6}$ \\ Martijn A Spruit $\mathbb{D}^{5,7,8}$ \\ Ulla Møller Weinreich (DD ${ }^{1,2}$ \\ 'Department of Respiratory Diseases, \\ Aalborg University Hospital, Aalborg, \\ Denmark; ${ }^{2}$ The Clinical Institute, Aalborg \\ University, Aalborg, Denmark; \\ ${ }^{3}$ Department of Cardiology, Aalborg \\ University Hospital, Aalborg, Denmark; \\ ${ }^{4}$ Department of Clinical Research, \\ Hillerød Hospital, Hillerød, Denmark; \\ ${ }^{5}$ Department of Research and \\ Development, Ciro, Horn, the \\ Netherland; ${ }^{6}$ Department of Health \\ Services Research, Care and Public \\ Health Research Institute, Faculty of \\ Health, Medicine and Life Sciences, \\ Maastricht University, Maastricht, the \\ Netherlands; ${ }^{7}$ Nutrim School of \\ Nutrition and Translational Research in \\ Metabolism, Faculty of Health, Medicine \\ and Life Sciences, Maastricht University, \\ Maastricht, the Netherlands; \\ ${ }^{8}$ Department of Respiratory Medicine, \\ Maastricht University Medical Centre \\ (MUMC+), Maastricht, the Netherlands
}

Correspondence: Peter Ascanius Jacobsen Department of Respiratory Diseases, Aalborg University Hospital, Aalborg, Denmark

Tel +4597664800

Email peter.jacobsen@rn.dk
Introduction: The understanding of whether and to what extent employment status affects readmission and mortality is limited in patients with COPD.

Aim: To explore how employment status affects readmission and mortality after first admission to the hospital with acute exacerbation of COPD (AECOPD).

Methods: This study used Danish national registry-based data. All patients admitted for the first time to the hospital between 1999 and 2014 with a diagnosis of AECOPD, age 35-59, without a previous asthma diagnosis were included in the study. Employment status effect on 30-, 90-, and 365-day readmission and mortality was examined using logistic regression, adjusting for relevant confounders.

Results: A total of 11,850 COPD patients were included in the study of which $3563(30 \%)$ were working, 1368 (12\%) unemployed, 840 (7\%) on sick leave, and 6079 (51\%) receiving early retirement. Patients receiving early retirement had, compared to patients working, an adjusted increased likelihood of readmission at 30, 90, and 365 days (odds ratio (OR) 1.26 $\left(\mathrm{CI}^{95 \%}(1.06-1.49)\right), 1.33\left(\mathrm{CI}^{95 \%}(1.16-1.53)\right)$, and $1.48\left(\mathrm{CI}^{95 \%}(1.33-1.66)\right)$, respectively). An increased likelihood was also seen in unemployed at 365 days follow-up (OR $1.44\left(\mathrm{CI}^{95 \%}\right.$ $(1.22-1.68)))$. Early retirement was associated with an increased mortality at 30,90 , and 365 days (OR $1.39\left(\mathrm{CI}^{95 \%}(1.07-1.80)\right) 1.37\left(\mathrm{CI}^{95 \%}(1.09-1.79)\right)$ and $1.48\left(\mathrm{CI}^{95 \%}(1.25-1.75)\right)$, respectively). An increased likelihood was also seen in patients receiving sick leave (OR $\left.1.57\left(\mathrm{CI}^{95 \%}(1.21-2.04)\right)\right)$.

Conclusion: Patients with COPD who are not working at the time of first admission have a higher likelihood of readmission and mortality.

Keywords: chronic obstructive pulmonary disease, employment, mortality, readmission

Chronic obstructive pulmonary disease (COPD) is the third leading cause of death. ${ }^{1}$ Mortality is often seen in relation to acute exacerbations of COPD (AECOPD), ${ }^{2}$ not least severe AECOPD ${ }^{3}$ ie, AECOPD leading to hospitalization. Mortality rates following AECOPD vary from $3.6 \%$ in short-term mortality to $31 \%$ in two-year mortality ${ }^{4}$ and the risk of mortality increases with readmission after AECOPD. ${ }^{5}$ Readmission rates after hospitalization for AECOPD within 30 days range from 8.8 to $26.0 \%$ and for 90-day readmissions from 17.5 to $39.0 \% .{ }^{6}$ Higher readmission rates are seen in patients with comorbidities, male sex, previous admissions and exacerbations and longer hospital admission. ${ }^{6}$ Increased mortality is seen in patients with sedentary lifestyle, suggesting that an active lifestyle is important for patients. ${ }^{7,8}$

Patients with COPD are often outside the workforce and experience activity limitations as a consequence of COPD. ${ }^{9-11}$ Studies have explored the effects of retirement on physical activity with ambiguous findings of increased leisure activity 
but also more sedentary behavior. ${ }^{12}$ For patients with COPD outside the workforce, lower exercise capacity and daily physical activity have been found compared to patients with COPD who are working, as explored in a study performed on a Dutch population (submitted by Peter et al).

Disability pension, as reported by Tøttenborg et al, comes with an increased risk of exacerbation, admission, and death in COPD-patients.${ }^{13}$ Unemployment was explored in the same study, but this was not associated with an increased risk of admission and death; interestingly, it even seemed to be beneficial in terms of reducing AECOPD. The study did, however, not focus specifically on patients of working age (ie, 18-65 years of age), nor was connection to the workforce the main target of this study. As such, our knowledge of the effects of employment status on readmission and mortality after severe AECOPD is sparse. By exploring this we may be able to identify a potentially vulnerable subgroup within the COPD-population, where early intervention could change the clinical outcomes.

The aim of the study was therefore to explore how employment status is associated with short- (30 days), medium- (90 days) and long- (365 days) term readmission and mortality following severe AECOPD in patients with COPD within working age. We hypothesized a priori that patients working have lower readmission and mortality risk than patients without work, also after correction for confounding variables.

\section{Method}

\section{Study Setting}

All Danish citizens have access to free health care and public support in the form of unemployment benefit, sick leave benefit (available after 3 weeks of sick leave), and early retirement benefit (available for people with significant impaired workability). Through the Danish civil personal registration number (CPR), unique to all citizens, connection between different national registers is possible.

\section{Data Sources}

This study uses nationwide registry data: The National Patient Registry (LPR) and the National Psychiatric Registry to identify patients and patients' comorbidities through International Statistical Classification of Diseases and Related Health Problems, version 10 (ICD-10) diagnosis codes, related to hospital admissions and visits; the
National Labor Market Database (DREAM) to identify patients receiving public financial support; the National Prescription Registry for medication use; the Danish Cause of Death registry to identify date of death from and the Statistics of Denmark Registry to identify patients' age, sex and education level.

Retrospective registry studies do not require ethical approval in Denmark. The data responsible institute (The Capital Region of Denmark) approved access to data for the purpose of this project (project id: P2019-402).

\section{Study Population}

Patients between 35 and 59 years were included in their first ever admission to the hospital with AECOPD between 1999 and 2014 with a primary diagnosis of COPD (DJ44) or a primary diagnosis of respiratory failure (DJ96) or pneumonia (DJ18) and a secondary diagnosis of COPD. ${ }^{14}$ Patients admitted with the above-mentioned criteria prior to 1999 were not included. These criteria have been validated as hospital admission of AECOPD in the Danish registry with a positive predictive value of $92 \%{ }^{14}$ Admissions with AECOPD were defined as patients admitted to a department where 24-hour surveillance of patients is possible. The age range was chosen because early voluntary retirement was available from the age of 60 during this time period which for the purpose of statistical analysis would make patients incomparable. Patients with asthmatic bronchitis or a previous diagnosis of asthma (DJ45) were excluded. Patients with incomplete data on cohabitation status and education level were also excluded.

\section{Outcome and Covariate Definitions}

Time from first admission to readmission with AECOPD as defined above, as well as time till death, was calculated. From this, 30, 90, and 365 days readmission and mortality were calculated. Employment status was categorized as either i) working, ii) unemployment benefit, iii) sick leave or iv) early retirement. Working included patients not receiving benefit (self-support), together with patients on certain public support characterized as work due to their time limited nature and an expectancy of work after support ends (eg, education benefit, maternity leave benefit). Self-support has a $98.2 \%$ predictive value in the DREAM registry. ${ }^{15}$ Sick leave was defined as sick leave three out of four weeks prior to admission, indicating longer sick leave periods, not necessarily associated with admission with AECOPD. Sick leave has a sensitivity of $96.7 \%$ in the DREAM registry. ${ }^{16}$ For further description of 
benefits see Table S1. Covariates included were age, sex, education level (short, medium, long and very long), cohabitation status, comorbidities (lung cancer, cancer, stroke, ischemic heart disease, heart failure, interstitial lung disease, atrial fibrillation/atrial flutter, chronic renal failure, diabetes, ulcer, anxiety, depression) and inhalation medication use (divided into the following exclusive categories: long acting muscarinic agonist (LAMA) + long acting $\beta 2$ agonist (LABA) + inhaled corticosteroid (ICS); LAMA +LABA; LAMA+ICS or LABA + ICS; LAMA; LABA; ICS; SABA only; no medication). Medication use was recorded for prescriptions redeemed 6 months prior to admission. The comorbidities anxiety, depression, and diabetes were located using both the prescription registry and LPR table, while the remaining comorbidities only were recorded from LPR (see Supplementary Material Table $\underline{\mathrm{S} 2}$ ). Diagnosis more than 5 years prior to admission was excluded to increase the relevance of comorbidities recorded.

\section{Statistical Analysis}

Data were, for continuous variables, reported using mean (standard deviation), when normally distributed, and as median (25 and 75 percentiles) when not normally distributed. Categorical variables are reported using count and percentages.

Cumulative incidence curves of readmission and mortality until one year after admission were depicted.

Logistic regression was used to examine 30-, 90-, and 365-day readmission and mortality including abovementioned covariates reporting odds ratios of employment status. Complete follow-up was available for both readmission and mortality with less than 4 patients had emigrated at one-year follow-up making censoring in the mortality analysis minimal. Patients dying during followup before readmission with AECOPD are presented in the results section, and the results regarding readmission must be interpreted with this in mind. Continuous variables were plotted for each value and their corresponding logit to assess the linearity assumption in logistic regression. As age data did not show a linear tendency across different ages, age was grouped into intervals (age groups: 35-45, 46-50, 51-55, and 56-59 years).

Data management and analyses were performed using SAS version 9.4 ("SAS Institute Inc., Cary, NC, USA") and $\mathrm{R}$ (version 4.0.3). A two-tailed p-value of 0.05 was considered significant.

\section{Results}

Out of 111,165 patients admitted for the first time with AECOPD, 12,377 patients were between 35 and 59 years old and had no previous asthma diagnosis. Of these 12,377 patients, 527 had missing data on education or cohabitation status and were excluded, leaving 11,850 patients available for analyses (see Figure 1).

Of all patients included, $30 \%(3,563 / 11,850)$ were supporting themselves and classified as working, 12\% (1,368/ $11,850)$ were receiving unemployment benefit, $7 \%(840 /$ $11,850)$ were receiving sick leave benefit, and 51\% $(6,079 /$ $11,850)$ were receiving early retirement benefit. Patient characteristics in subgroups of employment status are shown in Table 1. Patients employed had higher education level, were not living alone, had fewer comorbidities and less not receiving medication use compared some or all the other subgroups. Higher rates of comorbidities are seen in patients receiving sick leave or early retirement benefit (eg, cancer, heart failure, ischemic heart disease, stroke) compared to patients working or unemployed (see Table 1).

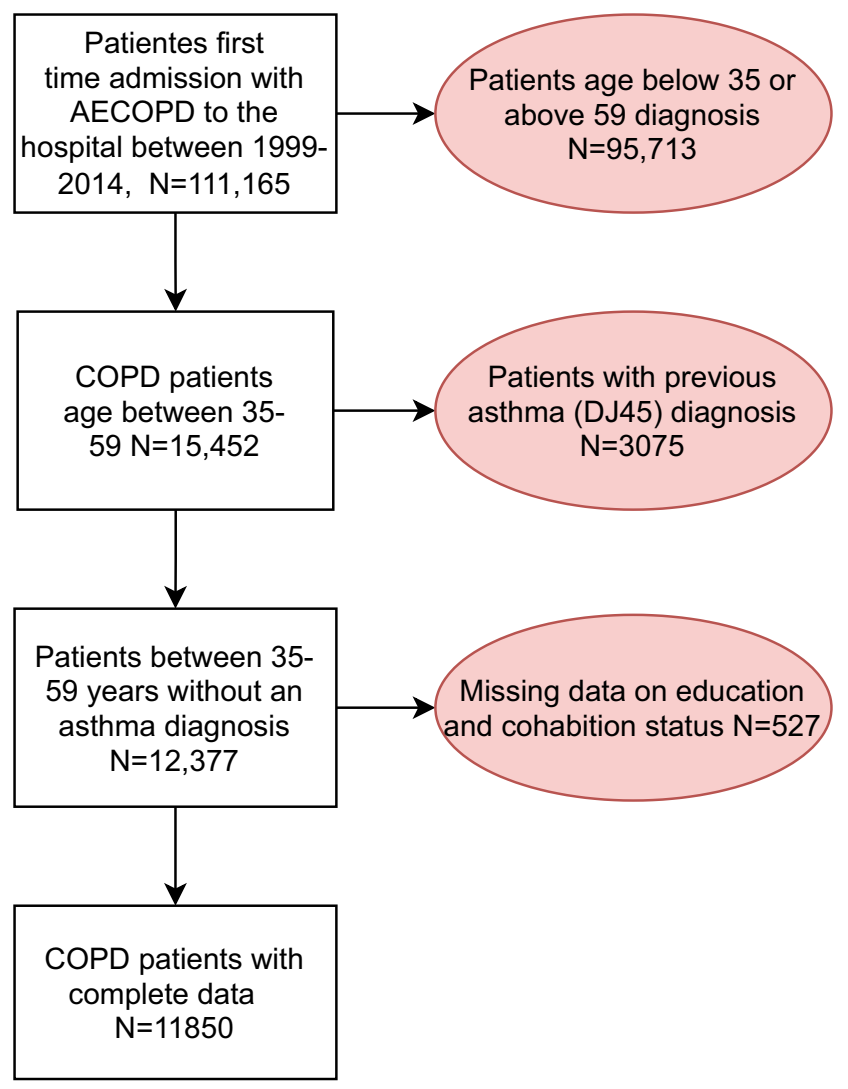

Figure I Inclusion of AECOPD patients. Red boxes show excluded patients. 
Table I Demographics of Patients at First Admission with AECOPD

\begin{tabular}{|c|c|c|c|c|c|}
\hline Variable & Level & $\begin{array}{l}\text { Working } \\
(n=3563)\end{array}$ & $\begin{array}{l}\text { Unemployed } \\
(n=1368)\end{array}$ & $\begin{array}{l}\text { Sick Leave } \\
(n=840)\end{array}$ & $\begin{array}{l}\text { Early Retirement } \\
(n=6079)\end{array}$ \\
\hline Sex & Male & I65I (46.3) & $720(52.6)$ & $421(50.1)$ & $2775(45.6)$ \\
\hline Age & Median (IQR) & $54[50,57]$ & $52[47,56]$ & $54[50,57]$ & $55[51,57]$ \\
\hline \multirow[t]{4}{*}{ Age group } & $35-45$ & $446(12.5)$ & $282(20.6)$ & $90(10.7)$ & $455(7.5)$ \\
\hline & $46-50$ & $566(15.9)$ & $268(19.6)$ & $132(15.7)$ & 931 (I5.3) \\
\hline & $5 I-55$ & 1168 (32.8) & $4 I I(30.0)$ & $303(36.1)$ & $1927(31.7)$ \\
\hline & $56-59$ & I383 (38.8) & $407(29.8)$ & $315(37.5)$ & $2766(45.5)$ \\
\hline \multirow[t]{3}{*}{ Educational level } & Short & $\mathrm{I}, 455(40.8)$ & $756(55.3)$ & $358(42.6)$ & $3,898(64.1)$ \\
\hline & Medium & $1633(45.8)$ & $499(36.5)$ & $37 \mid(44.2)$ & $1796(29.5)$ \\
\hline & Long/Very long & $475(13.3)$ & $113(8.3)$ & III (I3.2) & $385(6.3)$ \\
\hline Living alone & & $1170(32.8)$ & $803(58.7)$ & $325(38.7)$ & $3621(59.6)$ \\
\hline Lung cancer & & $30(0.8)$ & $13(1.0)$ & $38(4.5)$ & $135(2.2)$ \\
\hline Cancer & & $92(2.6)$ & $29(2.1)$ & $59(7.0)$ & $275(4.5)$ \\
\hline Interstitial lung disease & & $47(1.3)$ & $15(1.1)$ & $37(4.4)$ & $|2|(2.0)$ \\
\hline AF/AFL & & $90(2.5)$ & $23(1.7)$ & $53(6.3)$ & 240 (3.9) \\
\hline Heart Failure & & $148(4.2)$ & $55(4.0)$ & $70(8.3)$ & $553(9.1)$ \\
\hline Ischaemic heart disease & & $238(6.7)$ & $106(7.7)$ & $101(12.0)$ & $718(11.8)$ \\
\hline Stroke & & $70(2.0)$ & $38(2.8)$ & $48(5.7)$ & $415(6.8)$ \\
\hline Ulcer & & $74(2.1)$ & $48(3.5)$ & $23(2.7)$ & $285(4.7)$ \\
\hline Chronic renal failure & & $23(0.6)$ & $13(1.0)$ & $13(1.5)$ & $149(2.5)$ \\
\hline Diabetes & & $216(6.1)$ & $87(6.4)$ & $77(9.2)$ & $779(12.8)$ \\
\hline Anxiety & & $69(1.9)$ & $64(4.7)$ & $46(5.5)$ & $210(3.5)$ \\
\hline Depression & & $114(3.2)$ & $72(5.3)$ & $96(11.4)$ & $327(5.4)$ \\
\hline \multirow[t]{4}{*}{ Comorbidity count } & 0 & $2,337(65.6)$ & $803(58.7)$ & $333(39.6)$ & $2,473(40.7)$ \\
\hline & 1 & $820(23.0)$ & $35 I(25.7)$ & $264(31.4)$ & $1786(29.4)$ \\
\hline & 2 & $268(7.5)$ & 147 (I0.7) & 140 (16.7) & $1004(16.5)$ \\
\hline & $3+$ & $138(3.9)$ & $67(4.9)$ & $103(12.3)$ & $816(13.4)$ \\
\hline \multirow[t]{7}{*}{ Inhalation medication } & No treatment & $\mathrm{I}, 180(33.1)$ & $576(42.1)$ & $274(32.6)$ & I,883 (31.0) \\
\hline & Triple Therapy & $334(9.4)$ & $108(7.9)$ & $139(16.5)$ & $919(15.1)$ \\
\hline & LAMA + ICS or LABA + ICS & $707(19.8)$ & $216(15.8)$ & $153(18.2)$ & $1224(20.1)$ \\
\hline & LAMA + LABA & $21(0.6)$ & $17(1.2)$ & $14(1.7)$ & $74(1.2)$ \\
\hline & LAMA or LABA & $240(6.7)$ & $83(6.1)$ & $59(7.0)$ & $413(6.8)$ \\
\hline & ICS only & $47 \mid(13.2)$ & $136(9.9)$ & $78(9.3)$ & $683(11.2)$ \\
\hline & SABA only & $610(17.1)$ & $232(17.0)$ & $123(14.6)$ & $883(14.5)$ \\
\hline Died at day of admission & & $27(0.8)$ & $21(1.5)$ & $12(1.4)$ & $130(2.1)$ \\
\hline
\end{tabular}

Notes: (\%) [25 and 75 percentile]. Long and very long education level are displayed as one group to avoid low numbers in columns in accordance with Danish law.

Abbreviations: AF, atrial fibrillation; AFL, atrial Flutter; LAMA, long acting muscarinic antagonist; LABA, long acting beta2 agonist; ICS, inhaled corticosteroids; SABA, short acting beta2 agonist.

At first admission 33\% (3913/11,850) had not redeemed a prescription for obstructive airway medication 6 months prior. In this group, 58\% (2279/3913) had not before redeemed a prescription for obstructive airway medication when not restricting to 6 months accounting for $19 \%(2279 / 11,850)$ in the entire study. 
During the 30, 90, and 365-days follow-up 8\% (946/ $11,850), 12 \%(1478 / 11,850)$, and $23 \%(2,676 / 11,850)$ of the patients were readmitted, respectively. Mortality rates during the 30, 90, and 365-days follow-up were $4 \%$ (454/ $11,850), 5 \%(647 / 11,850)$, and $10 \%(1,222 / 11,850)$, respectively. Of the 1,222 that died during follow-up, 865 died before getting readmitted with AECOPD.

The cumulative incidence of readmission with AECOPD and mortality in the different workforce strata are shown in Figures 2 and 3. The lowest rate of readmission is seen in patients working, with similar higher rates in patients receiving sick leave or unemployment benefit and with the highest rates seen in patients receiving early retirement. The lowest rate of mortality is seen in patients working with slightly higher rates of unemployment and the highest rates in patients receiving sick leave or early retirement benefit.

The results of the logistic regression analysis regarding employment status are shown in Figure 4 with the full models shown in Figures S1-S6. Significantly higher likelihoods for readmission were seen at 30-, 90-, and 365-day follow-up in patients receiving early retirement and also at 365-day follow-up for patients receiving unemployment benefit compared to patients working adjusting for relevant confounders.

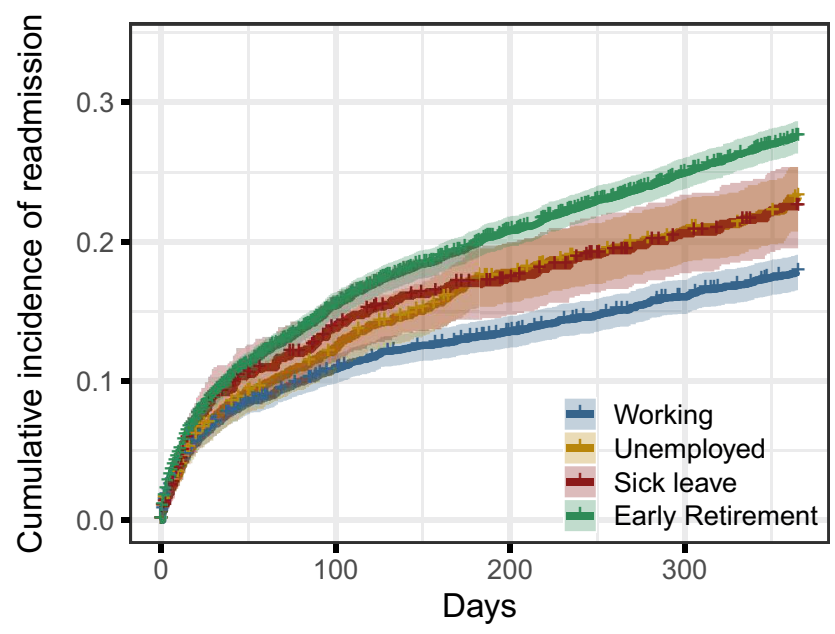

Number at risk

$=$\begin{tabular}{llcc|}
3563 & 3084 & 2971 & 2865 \\
1368 & 1155 & 1068 & 1016 \\
840 & 677 & 634 & 591 \\
6079 & 4831 & 4438 & 4143 \\
\hline
\end{tabular}

Figure 2 Cumulative incidence curves for readmission. At risk table display COPD patients who has not been readmitted or died.

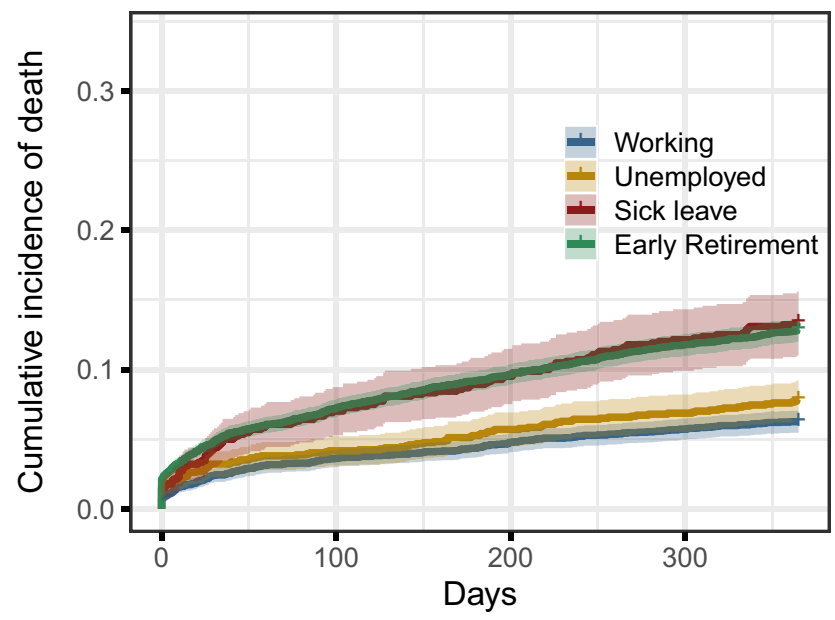

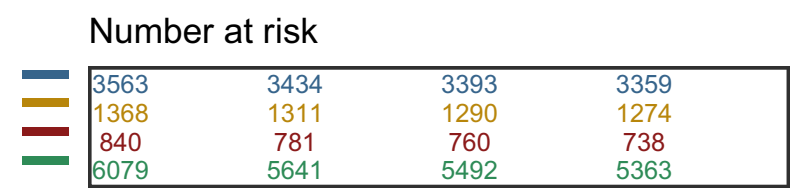

Figure 3 Cumulative incidence curves for mortality. At risk table display COPD patients who has not died.

Significantly higher likelihoods for mortality were seen at 30-, 90-, and 365-day follow-up in patients receiving early retirement and also at 365-day follow-up for patients receiving sick leaves compared to patients working adjusting for relevant confounders.

\section{Discussion}

This nationwide study of AECOPD patients from Denmark, between year 1999-2014, found a higher likelihood of readmission and mortality primarily in patients receiving early retirement benefit at day 30,90 and 365 after admission due to AECOPD. Patients receiving unemployment benefit had a higher risk of readmission at 365day follow-up, and patients receiving sick leave benefit had a higher mortality at 365-day follow-up.

This study contributes to a better understanding of the impact of patients' employment status at first admission on patients' outcomes with regard to readmission and mortality. An increased risk of admission and mortality in patients receiving disability pension (characterized as early retirement in this study) has previously been described by Tøttenborg et al. ${ }^{13}$ Our study supports these findings in patients receiving early retirement benefit in a large population where patients at inclusion are too young to receive early voluntary retirement and regular pension. A one-year mortality rate of $11 \%$ was seen in the 


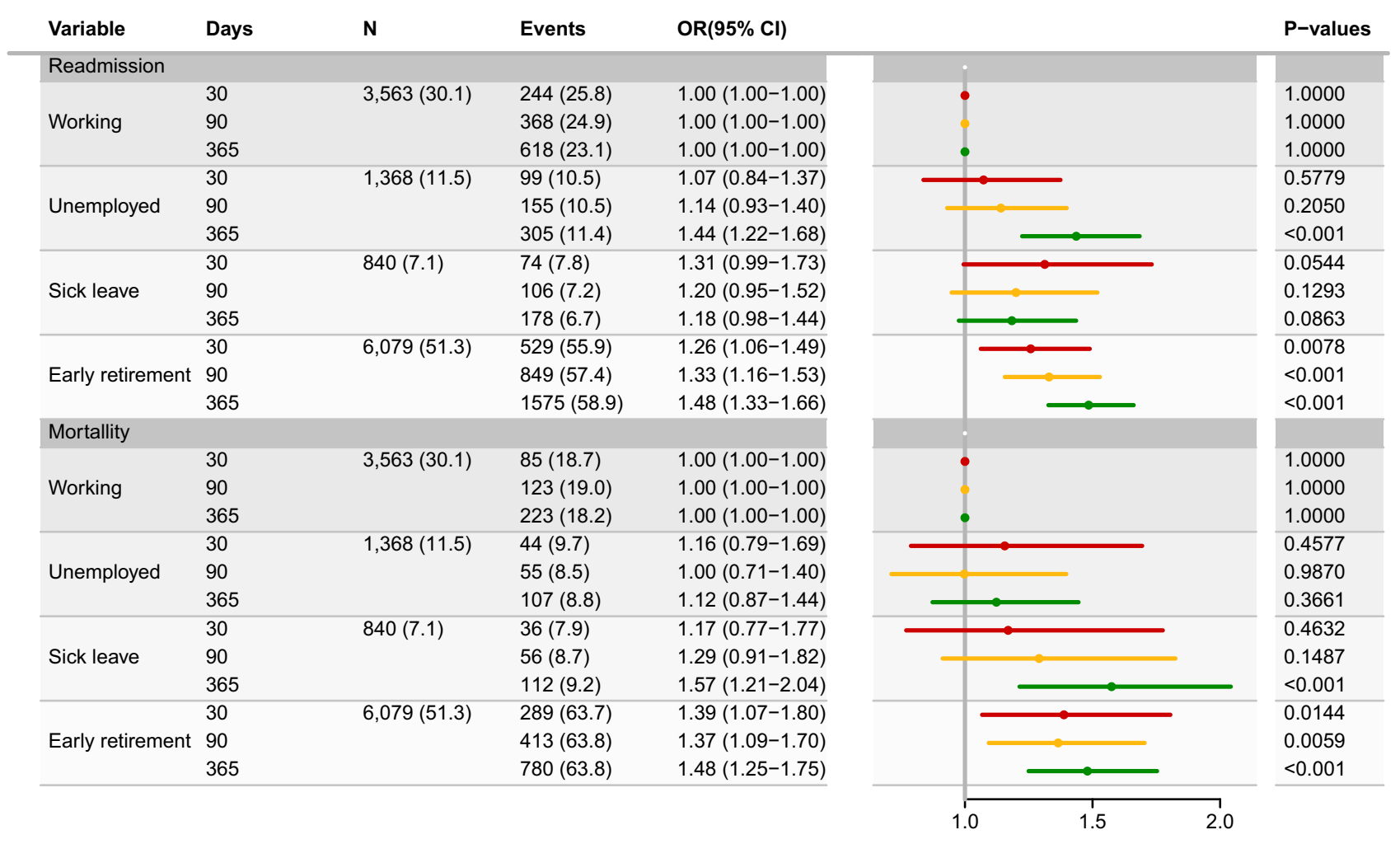

Figure 4 Multivariable logistic regression of 30-, 90- and 365-days' readmission and mortality following first admission with AECOPD. 30-, 90- and 365-days mortality is indicated by red, yellow and green respectively. Output in figure is adjusted for age, sex, education, cohabitation status, lung cancer, other cancer, pulmonary fibrosis, atrial fibrillation/atrial flutter, heart failure, ischemic heart disease, cerebrovascular disease, gastric ulcer, chronic kidney failure, diabetes, anxiety, depression and inhalation medication combination.

Tøttenborg et al study which included all age groups. A relatively high short-term mortality is seen at first admission in our much younger group of $4-5 \%$ at $30-90-$ day mortality and $10 \%$ within one year. Unemployment was associated with an increased risk of readmission, inconsistent with the finding of the previously mentioned work by Tøttenborg et al. Furthermore, a tendency towards increased likelihood of admission is seen in patients receiving sick leave at first admission. This distinction was not explored in the study by Tøttenborg et al which may also explain the differences seen since these patients are likely to have been classified as working. The increased likelihood of mortality after one year in these patients underlines that patients at first admission are a group of concern. It may be that differences in risk of readmission seen in the study are related to our study including patients at first admission instead of at contact with an outpatient clinic. This timepoint may include more vulnerable patients together with a higher short-term risk of readmission and mortality, making our study population more vulnerable even though we only include younger patients. First admission is therefore not only a major lifeevent but also a time point of interest in order to improve patients' outcomes, and assessment of workforce connection may help identify the more vulnerable patients.

The patients who receive early retirement may have different reasons for not being able to work. The benefit is only available to people who have impaired capability to work. In a population of patients with COPD, it is likely that some of this impairment comes from the symptoms such as dyspnea and fatigue related to COPD. Other conditions may, however, contribute as indicated in the distribution of comorbidities between employment groups. This raises the question of whether patients leave the workforce due to COPD or whether patients' comorbid conditions play a role in the detachment and increased mortality. Since the main cause of COPD in Denmark is tobacco smoking, it is likely that other conditions, such as cardiovascular diseases and malignancy, which are more frequent in patients receiving sick leave and early retirement, are contributing to the mortality in the population and also to the overall burden of morbidity that leads to 
workforce detachment. By adjusting for multiple different relevant comorbidities, this study still reveals an association between employment status and mortality.

COPD patients without paid work have previously been shown to have lower physical activity and lower exercise capacity compared to patients with paid work. ${ }^{17}$ Some of the negative effects seen in patients receiving sick leave and early retirement benefit may therefore be related to patient's activity level. Lower physical activity and lower exercise capacity are associated with a higher risk of mortality and readmission in patients with COPD..$^{8,18-21}$ Maintaining employment may be a way to avoid a more sedentary lifestyle and ultimately improve patient outcomes. On the other hand, as mortality in our study is high after AECOPD in this young cohort, it also calls for an increased focus on patients in need of advance care planning and palliative care. Being unable to work may help guide clinicians in recognizing patients with a poorer prognosis and palliative care needs.

This study shows that more than half of the patients have already left the workforce at their first AECOPD admission. An average age of early retirement has previously been estimated at 54 years, and most patients are diagnosed COPD after age $40 .^{22}$ Intervention should therefore be considered at the time of diagnosis to avoid workforce detachment and the consequences regarding readmission and mortality related to this. Another area of concern is in patients who at this relatively young age are admitted for the first time and on sick leave, unemployed or reviving early retirement. Intervention such as pulmonary rehabilitation following admission may help these patients understand the importance of having an active lifestyle, improving their physical ability, and thereby improving their readmission rates and mortality.

This study has some limitations. Due to the registrybased retrospective study design, the causal effects may be explained in multiple ways as described above. The two major causal effects being: Does COPD lead to workforce detachment or does workforce detachment increase the risk and negative outcomes of COPD? Patients' reasons for leaving the workforce were unfortunately not available. This may have improved understanding the mechanisms leading to increased readmission rates and mortality.

Clinical measurements were, due to the registrybased study design, unfortunately not available. Information on, eg, lung function, disease severity, quality of life measurement, physical activity and smoking status may have improved our logistic regression models and supported the understanding of how work status affect readmission and mortality. The closest proxy measurement of disease severity is inhalation medication treatment step, which in the analysis indicated a higher likelihood of readmission, with comorbidities not affecting readmission rates with the exception of interstitial lung diseases. Inhalation medication, however, indicates slight benefits in the analysis of mortality with multiple comorbidities having highly significant effects on patient's mortality. This could indicate that the majority of patients that die in this age group suffer from multiple diseases, which the data also indicate with $50 \%$ of the study population having zero comorbidities and this group only accounting for 315 of the 1222 who die.

Time of detachment was not explored, and some patients may have left the workforce before the development of COPD. The number is unknown, not least due to the DREAM database, which is used to classify workforce connection, only contains data from 1992 onwards. This knowledge would have helped understand the magnitude of patients where an intervention might be possible.

One-third of the population did not receive inhaled medication at first admission. Of those, almost half the patients had previously redeemed inhaled medication and it is therefore likely that they are non-adherent to medication, as many COPD patients are. ${ }^{23}$ The remainder may of course be mis-classified, however, previous studies found higher numbers of patients diagnosed with COPD at first admission. $^{24,25}$ We therefore believe this cohort to be representative of a first-time admitted AECOPD-cohort.

\section{Interpretation}

This study shows that COPD-patients receiving sick leave, unemployment and early retirement benefit have an increased risk of readmission and mortality within one year. Workforce detachment may be an early indicator in COPD patients with a poor prognosis; however, a better understanding of why patients outside the workforce have a poor prognosis is needed.

\section{Acknowledgment}

All authors contributed to designing the study, interpreting the data, drafting and editing the manuscript. Data was available through Christian Torp-Pedersen who assisted in the relevant approval that made the study possible. Peter Jacobsen had the primary responsibility in drafting the manuscript with Ulla Møller Weinrich as the primary supervisor. 


\section{Funding}

There was no funding obtained in the execution of this study.

\section{Disclosure}

Dr Kristian Hay Kragholm reports personal fees from Novartis, outside the submitted work. Dr Christian TorpPedersen reports grant for randomised study from Bayer and grant for epidemiological study from Novo Nordisk, during the conduct of the study. Dr Daisy JA Janssen reports lecture fees from Novartis and Boehringer Ingelheim, outside the submitted work. The authors have no other conflicts of interest to declare with regard to the content of this manuscript.

\section{References}

1. World Health Organization. The top 10 causes of death; 2016. Available from: https://www.who.int/news-room/fact-sheets/detail/ the-top-10-causes-of-death. Accessed November 6, 2020.

2. Schmidt SA, Johansen MB, Olsen M, et al. The impact of exacerbation frequency on mortality following acute exacerbations of COPD: a registry-based cohort study. BMJ Open. 2014;4(12):e006720. doi:10.1136/bmjopen-2014-006720

3. Hoogendoorn M, Hoogenveen RT, Rutten-van Molken MP, Vestbo J, Feenstra TL. Case fatality of COPD exacerbations: a meta-analysis and statistical modelling approach. Eur Respir J. 2011;37 (3):508-515. doi:10.1183/09031936.00043710

4. Singanayagam A, Schembri S, Chalmers JD. Predictors of mortality in hospitalized adults with acute exacerbation of chronic obstructive pulmonary disease. A systematic review and meta-analysis. Ann Am Thorac Soc. 2013;10(2):81-89. doi:10.1513/AnnalsATS.201208-043OC

5. Hartl S, Lopez-Campos JL, Pozo-Rodriguez F, et al. Risk of death and readmission of hospital-admitted COPD exacerbations: European COPD audit. Eur Respir J. 2016;47(1):113-121. doi:10.1183/ 13993003.01391-2014

6. Alqahtani JS, Njoku CM, Bereznicki B, et al. Risk factors for all-cause hospital readmission following exacerbation of COPD: a systematic review and meta-analysis. Eur Respir Rev. 2020;29 (156):190166. doi:10.1183/16000617.0166-2019

7. Spruit MA, Pitta F, McAuley E, ZuWallack RL, Nici L. Pulmonary rehabilitation and physical activity in patients with chronic obstructive pulmonary disease. Am J Respir Crit Care Med. 2015;192 (8):924-933. doi:10.1164/rccm.201505-0929CI

8. Waschki B, Kirsten A, Holz O, et al. Physical activity is the strongest predictor of all-cause mortality in patients with COPD: a prospective cohort study. Chest. 2011;140(2):331-342. doi:10.1378/chest.10-2521

9. Wheaton AG, Cunningham TJ, Ford ES, Croft JB. Employment and activity limitations among adults with chronic obstructive pulmonary disease - United States, 2013. MMWR Morb Mortal Wkly Rep. 2015;64(11):289-295.

10. Rai KK, Adab P, Ayres JG, Jordan RE. Systematic review: chronic obstructive pulmonary disease and work-related outcomes. Occup Med. 2018;68(2):99-108. doi:10.1093/occmed/kqy012

11. Rai KK, Jordan RE, Siebert WS, et al. Birmingham COPD cohort: a cross-sectional analysis of the factors associated with the likelihood of being in paid employment among people with COPD. Int J COPD. 2017;12:233-242. doi:10.2147/COPD.S119467
12. Xue B, Head J, McMunn A, Heyn PC. The impact of retirement on cardiovascular disease and its risk factors: a systematic review of longitudinal studies. Gerontologist. 2020;60(5):e367-e377. doi: 10.109

3/geront/gnz062

13. Tottenborg SS, Lange P, Johnsen SP, Nielsen H, Ingebrigtsen TS, Thomsen RW. Socioeconomic inequalities in adherence to inhaled maintenance medications and clinical prognosis of COPD. Respir Med. 2016;119:160-167. doi:10.1016/j.rmed.2016.09.007

14. Thomsen RW, Lange P, Hellquist B, et al. Validity and underrecording of diagnosis of COPD in the Danish national patient registry. Respir Med. 2011;105(7):1063-1068. doi:10.1016/j.rmed.20 11.01 .012

15. Hjollund NH, Larsen FB, Andersen JH. Register-based follow-up of social benefits and other transfer payments: accuracy and degree of completeness in a Danish interdepartmental administrative database compared with a population-based survey. Scand J Public Health. 2007;35(5):497-502. doi:10.1080/14034940701271882

16. Stapelfeldt CM, Jensen C, Andersen NT, Fleten N, Nielsen CV. Validation of sick leave measures: self-reported sick leave and sickness benefit data from a Danish national register compared to multiple workplace-registered sick leave spells in a Danish municipality. BMC Public Health. 2012;12:661. doi:10.1186/1471-2458-12-661

17. Jacobsen PA, van 'T Hul AJ, Djamin RS, et al. Characteristics and treatable traits of patients with chronic obstructive pulmonary disease (COPD) with and without paid employment. Respir Res. 2021;22 (1):147. doi:10.1186/s12931-021-01736-6

18. Liu Y, Croft JB, Anderson LA, Wheaton AG, Presley-Cantrell LR, Ford ES. The association of chronic obstructive pulmonary disease, disability, engagement in social activities, and mortality among US adults aged 70 years or older, 1994-2006. Int J Chron Obstruct Pulmon Dis. 2014;9:75-83. doi:10.2147/COPD.S53676

19. Vaes AW, Garcia-Aymerich J, Marott JL, et al. Changes in physical activity and all-cause mortality in COPD. Eur Respir J. 2014;44 (5):1199-1209. doi:10.1183/09031936.00023214

20. Nguyen HQ, Chu L, Amy liu IL, et al. Associations between physical activity and 30-day readmission risk in chronic obstructive pulmonary disease. Ann Am Thorac Soc. 2014;11(5):695-705. doi:10.1513/ AnnalsATS.201401-017OC

21. Bernabeu-Mora R, Garcia-Guillamon G, Valera-Novella E, GimenezGimenez LM, Escolar-Reina P, Medina-Mirapeix F. Frailty is a predictive factor of readmission within 90 days of hospitalization for acute exacerbations of chronic obstructive pulmonary disease: a longitudinal study. Ther Adv Respir Dis. 2017;11(10):383-392. doi: $10.1177 / 1753465817726314$

22. Fletcher MJ, Upton J, Taylor-Fishwick J, et al. COPD uncovered: an international survey on the impact of chronic obstructive pulmonary disease [COPD] on a working age population. BMC Public Health. 2011;11(1):612. doi:10.1186/1471-2458-11-612

23. Ingebrigtsen TS, Marott JL, Vestbo J, et al. Characteristics of undertreatment in COPD in the general population. Chest. 2013;144 (6):1811-1818. doi:10.1378/chest.13-0453

24. Balcells E, Anto JM, Gea J, et al. Characteristics of patients admitted for the first time for COPD exacerbation. Respir Med. 2009;103 (9):1293-1302. doi:10.1016/j.rmed.2009.04.001

25. Bastin AJ, Starling L, Ahmed R, et al. High prevalence of undiagnosed and severe chronic obstructive pulmonary disease at first hospital admission with acute exacerbation. Chron Respir Dis. 2010;7(2):91-97. doi:10.1177/1479972310364587 


\section{Publish your work in this journal}

The International Journal of COPD is an international, peer-reviewed journal of therapeutics and pharmacology focusing on concise rapid reporting of clinical studies and reviews in COPD. Special focus is given to the pathophysiological processes underlying the disease, intervention programs, patient focused education, and self management

protocols. This journal is indexed on PubMed Central, MedLine and CAS. The manuscript management system is completely online and includes a very quick and fair peer-review system, which is all easy to use. Visit http://www.dovepress.com/testimonials.php to read real quotes from published authors.

Submit your manuscript here: https://www.dovepress.com/international-journal-of-chronic-obstructive-pulmonary-disease-journal 